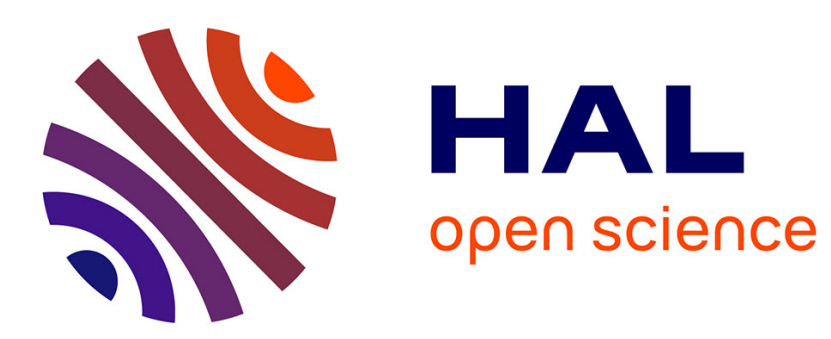

\title{
Successful treatment of drug-resistant cataplexy with the anticholinergic drug tropatepine
} Milan Nigam, Smaranda Leu-Semenescu, Isabelle Arnulf

\section{To cite this version:}

Milan Nigam, Smaranda Leu-Semenescu, Isabelle Arnulf. Successful treatment of drug-resistant cataplexy with the anticholinergic drug tropatepine. Journal of Clinical Sleep Medicine, 2021, 17 (4), pp.849-851. 10.5664/jcsm.9030 . hal-03478818

\section{HAL Id: hal-03478818 https://hal.sorbonne-universite.fr/hal-03478818}

Submitted on 14 Dec 2021

HAL is a multi-disciplinary open access archive for the deposit and dissemination of scientific research documents, whether they are published or not. The documents may come from teaching and research institutions in France or abroad, or from public or private research centers.
L'archive ouverte pluridisciplinaire HAL, est destinée au dépôt et à la diffusion de documents scientifiques de niveau recherche, publiés ou non, émanant des établissements d'enseignement et de recherche français ou étrangers, des laboratoires publics ou privés. 
Successful treatment of drug-resistant cataplexy with the anticholinergic drug tropatepine

Milan Nigam, MDa,b,c, Smaranda Leu-Semenescu, MDc, Isabelle Arnulf MD, PhDc,d

${ }^{a}$ Centre for Advanced Research in Sleep Medicine, Sacré-Coeur Hospital, Montreal, QC, Canada

${ }^{b}$ Department of Neurosciences, University of Montreal, Montreal, QC, Canada

c National Reference Center for Narcolepsy, Sleep Disorders Unit, University Hospital Pitié-Salpêtrière, Paris, France

a Sorbonne University, Paris, France

Keywords: narcolepsy, cataplexy, anticholinergic

Institution at which the work was performed:

Sleep Disorders Unit, Pitié-Salpêtrière University Hospital, Paris, France

Financial support:

No specific financial support was allocated to this project.

Manuscript submitted: Journal of Clinical Sleep Medicine

All authors have seen and approved the manuscript

Disclosure of conflicts of interest:

MN has received an educational grant from Paladin Labs pharmaceuticals.

SLS has speaking engagements with UCB Pharma.

IA has received consultant fees from Idorsia Pharma, Ono Pharma, UCB Pharma.

None of the financial disclosures is relevant to the submitted work.

This paper describes off-label use of the medication tropatepine

Abstract word count: 128

Manuscript word count: 2008, no figures or tables

Corresponding author:

Pr. Isabelle Arnulf, MD, PhD

Service des Pathologies du Sommeil, Hôpital Pitié-Salpêtrière

47-83 Bd de l'Hôpital, 75013 PARIS

Phone : $33(0) 1421677$ 02/04 
Fax : 33 (0) 142167700

E-mail: isabelle.arnulf@aphp.fr

Abbreviations

NT1: Narcolepsy type 1 


\section{ABSTRACT}

Narcolepsy type 1 is characterized by excessive daytime sleepiness and cataplexy, as well as hypocretin deficiency. Cataplexy (the loss of voluntary postural muscle tone, often in response to emotional stimuli), is one of the most disabling features, and is associated with significant social impairment and risk of injury. Cataplexy is usually alleviated by antidepressants, sodium oxybate and pitolisant. In this case report, we describe three patients with severe, drugresistant cataplexy, who experienced a dramatic improvement when treated with tropatepine, an anticholinergic muscarinic antagonist (which is commonly used to prevent neuroleptic-induced parkinsonism) after having failed usual treatments. The single side effect was mild mouth dryness. In addition to providing a new therapeutic option for resistant cataplexy, this benefit supports a role of cholinergic muscarinic transmission in REM sleep atonia.

\section{INTRODUCTION}

Narcolepsy type 1 (NT1) is a neurological disorder caused by the loss of hypocretin-1 neurons in the lateral hypothalamus. It is characterized by excessive daytime sleepiness, sleep attacks and cataplexy, as well as other variably present symptoms, including sleep paralysis, hypnagogic hallucinations, disturbed nighttime sleep, rapid eye movement (REM) sleep behavior disorder and weight gain. ${ }^{1}$ Cataplexy corresponds to brief loss of bilateral postural voluntary muscle tone, usually triggered by emotional stimuli. It is conceptualized as the intrusion of REM sleep atonia into wakefulness. Attacks can be either partial (i.e., loss of facial tone, dysarthria) or complete, involving the whole body and resulting into falls. Both types of cataplexy are a source of significant social impairment, anxiety and potential injury. Current treatment options for cataplexy include antidepressants (such as venlafaxine, paroxetine, escitalopram, fluoxetine and clomipramine), sodium oxybate and pitolisant.1,2 Although these agents are usually efficacious, some patients report persistent cataplexy despite multiple drug trials. This report describes three such patients, who experienced dramatic improvement following treatment with tropatepine, an anticholinergic muscarinic antagonist, primarily used in France as a treatment for neuroleptic-induced parkinsonism. ${ }^{3}$ In addition to its practical therapeutic 
implication, the successful use of this treatment re-emphasizes the importance of cholinergic mechanisms in the neurobiology of cataplexy.

\section{CASE REPORTS}

\section{Patient \#1}

A 27-year-old man was diagnosed with NT1 at age ten following a typical history of rapid weight gain, followed by progressive onset of excessive daytime sleepiness and cataplexy. The hypocretin- 1 level was undetectable $(<40$ $\mathrm{mg} / \mathrm{mL}$ ) in the CSF. He received intravenous immunoglobulins 5 months after cataplexy onset, which led to a decrease of total and partial cataplexy, which gradually reoccurred several months after the infusion. ${ }^{4}$ Over the subsequent years, the patient was treated with several wake-promoting agents with variable success, including modafinil, sodium oxybate and methylphenidate. The patient reported frequent, highly disabling partial cataplexy attacks characterized by loss of facial, neck and arm tone, provoked by laughter and lasting up to one minute. The attacks occurred dozens of times per day and were associated with significant social impairment and avoidance behaviours which had a profoundly negative impact on his quality of life. Previous trials of venlafaxine and sodium oxybate for cataplexy were inefficacious and the combination of pitolisant $36 \mathrm{mg}$ and clomipramine $25 \mathrm{mg}$ resulted in only a minor decrease in frequency of attacks. Methylphenidate, though effective for somnolence, triggered a severe psychotic episode at age 18, which was successfully treated with cessation of methylphenidate and introduction of risperidone, which was tapered over a twoyear period. To prevent neuroleptic-induced parkinsonism, tropatepine (Lepticur@, a muscarinic anticholinergic) was introduced and titrated up to $10 \mathrm{mg}$ daily. The dose was subsequently reduced to $5 \mathrm{mg}$ because of dry mouth. Within days of the introduction of tropatepine, the patient reported a dramatic reduction in the severity and frequency of cataplexy, with only minor, non-disabling facial cataplexy occurring once per week. He had since been treated with pitolisant $36 \mathrm{mg}$, and reported remarkable efficacy for daytime sleepiness. After one year on tropatepine, the patient attempted a one-week tropatepine holiday (but still took pitolisant) which caused the cataplexy to return to its pretreatment severity, which remitted once again upon resumption of tropatepine. The patient had now been on 
tropatepine for seven years, and has enjoyed continued efficacy. Importantly, he also reported a significant reduction in social anxiety related to his cataplexy, which has allowed him to pursue fulfilling relationships and career opportunities previously impossible.

\section{Patient \#2}

A 54-year-old man initially presented excessive daytime sleepiness in his early twenties, followed by facial cataplexy, which began near the age of thirty. The patient also exhibited occasional dream enactment behaviour compatible with REM sleep behaviour disorder (rarely, these had resulted in minor punches of his wife). At the age of 37 , the patient sought out care and was diagnosed with NT1 following polysomnography and multiple sleep latency tests. He was treated with partial success with modafinil $500 \mathrm{mg}$ daily in two doses and paroxetine $40 \mathrm{mg}$. Past trials of methylphenidate and sodium oxybate were not tolerated. The patient was referred to our centre at the age of 52 years old for progressive worsening in severity of cataplexy over the previous year. At that time, he reported partial cataplexy up to fifty times daily, which was characterized by jaw weakness and dysarthria with head dropping. Full cataplexy, often resulting in complete falls occurred several times per week and lasted for up to two minutes. Some of these episodes occurred in dangerous situations, including while swimming and while climbing a ladder. Typical triggers were laughing, anger and surprise. However, the episodes had begun to occur spontaneously, particularly if he was feeling fatigued. The patient reported that his cataplexy was by far the most invalidating symptom of his narcolepsy, with significant impairment in social and occupational functioning resulting in invalidity from his previous employment as a factory worker. At this time, the patient was also diagnosed with severe obstructive sleep apnea (apnea-hypopnea index: 54/hour) which was successfully treated with continuous airway pressure therapy. For his cataplexy, the patient was treated with clomipramine titrated up to $75 \mathrm{mg}$ daily, replacing paroxetine. After several months of further worsening of cataplexy, paroxetine was re-introduced concomitantly with clomipramine. No improvement was noted over the following year. The patient reported frequent complete cataplexy episodes occurring multiple times daily with minor emotional triggers. At this point, tropatepine was introduced at $10 \mathrm{mg}$. The patient reported a rapid improvement in his cataplexy. At the most recent follow-up, 10 months after the introduction of tropatepine, he reported only 
occasional attacks of facial cataplexy, occurring up to three times per week. Interestingly, the patient's wife also reported a decrease in frequency of dream enactment behaviour, with only occasional somniloquy and no violent behaviours remaining. The patient did not report any modification in his daytime somnolence, which remained significant but was well managed with a programmed nap schedule. The treatment is well tolerated with the patient noting only mild mouth dryness.

\section{Patient \#3}

A 78-year-old woman had been diagnosed with late-onset narcolepsy at age 53. She presented with a typical history of excessive daytime somnolence with irrepressible sleep attacks followed by onset of facial cataplexy triggered by laughing. She was otherwise known to have degenerative disk disease, osteoarthritis of the hip requiring hip replacement, hypertension and hypothyroidism. She lived alone and remained fully independent in activities of daily living, with no significant cognitive impairment. After having been satisfactorily treated with a combination of modafinil and escitalopram for several years, the patient was referred to our tertiary sleep unit at the age of 74 for worsening cataplexy. In recent years, she had begun experiencing complete cataplectic attacks more frequently. She reported that when playing tag with her young grand-daughter, she would run around the table laughing with her grand-daughter in pursuit, only to be caught lying on the ground as her cataplexy overwhelmed her. These attacks, precipitated by strong laughter occurred several times per month. They had resulted on at least one occasion in a minor occipital trauma and the patient and her family were increasingly concerned with her risk of injury. She also reported frequent, bothersome nightmares, often culminating in hypnopompic hallucinations. Escitalopram was discontinued and the patient was successively treated over the next four years with clomipramine, followed by combined paroxetine and pitolisant, the latter of which replaced her modafinil. None of these treatments proved more than modestly effective for her cataplexy, though $18 \mathrm{mg}$ of pitolisant resulted in a significant improvement in her daytime sleepiness. Despite adherent use of pitolisant and paroxetine, the patient's cataplexy continued to worsen, and she reported multiple daily episodes of both partial and complete cataplexy. She also reported that the emotional threshold necessary to trigger cataplexy had become progressively lower, such that even mild bemusement could trigger attacks. Considering the significant risk for injury in this elderly patient, tropatepine was introduced at a dose of $5 \mathrm{mg}$. At last follow up, $2 \mathrm{months}$ 
after the initiation of the treatment, the patient reported complete resolution of both complete and partial cataplexy within one week of treatment initiation. Interestingly, she also reported complete resolution of both nightmares and hypnopompic hallucinations. The patient, who continues to live independently, reported no significant cognitive side effects but did complain of mild dryness of her mouth.

\section{Discussion}

To our knowledge, this is the first report of successful treatment of cataplexy with a specific anticholinergic drug. Although this class of drug has not commonly been employed in the treatment of cataplexy, its use is rational when one considers the important role acetylcholine plays in the genesis of both REM sleep and muscle atonia.

The critical of role of acetylcholine in REM sleep has been demonstrated in animal models dating back to the era REM sleep discovery. Jouvet demonstrated in his seminal works in cats that the infusion of carbachol (a potent cholinergic, muscarinic agonist) in the mediodorsal pontine tegmentum produces both the clinical and electrophysiological features of REM sleep, a finding later replicated in several feline and murine models. ${ }^{5,6}$ Moreover, administration of anticholinergic agents have been shown, though less consistently, to reverse these physiological features ${ }^{6}$ Recently, a loss of function model in knockout mice demonstrated that specific muscarinic receptors, Chrm1 and Chrm3, are necessary to generate REM sleep and provides further evidence supporting the essential role of acetylcholine in REM sleep. $^{7}$

Cataplexy is thought to represent the intrusion of REM sleep atonia into wakefulness, resulting from the abnormal activation of the subcoeruleus nucleus (an equivalent of the peri-locus coeruleus alpha in the cat and of the sublaterodorsalis nucleus in the rat) by overactive limbic structures. ${ }^{1,8}$ Data from canine studies suggest that this process is mediated in part by increased cholinergic tone. Mignot and colleagues have shown that injection of carbachol into the pontine reticular formation precipitates cataplexy in a dose dependant fashion in narcoleptic dogs. ${ }^{9} \mathrm{~A}$ similar response can be observed in non-narcoleptic dogs, though significantly higher doses are required. Taken together, these findings suggest that cholinoceptive hypersensitivity in the pontine reticular formation which, via its reciprocal 
connections to limbic structures, facilitate the pathological recruitment of REM atonia generating circuits in response to salient emotional stimuli.

Considering the abundant evidence demonstrating the importance of acetylcholine in cataplexy and REM sleep, it is logical to attempt treatment of cataplexy with specific anticholinergic agents. Indeed, the dramatic and prompt therapeutic response to the muscarinic antagonist tropatepine in our report of three patients with severe and drug resistant cataplexy lends credence to this approach. Additionally, although tricyclic antidepressants are thought to exert their efficacy in the treatment of cataplexy primarily through their monoaminergic properties, it is reasonable to hypothesize their substantial anticholinergic activity is a contributing factor. Interestingly, two of our patients exhibited other signs of REM sleep dysfunction, including REM sleep behaviour disorder and nightmares with hypnagogic hallucinations, respectively. The observed improvement in these symptoms with tropatepine, may be related to the REM sleep suppressing properties of anticholinergics. Tropatepine has a long half-life (40 hours), which may be an advantage compared to venlafaxine short half-life, which can result in rebound cataplexy when a single dose is forgotten..$^{10}$ Tropatepine is a common drug, marketed since 1975, which may induce atropinic side-effects (urine retention, constipation, blurred vision, rare confusion). The benefit to risk ratio was excellent in our patients, as side effect were limited to non-disturbing dry mouth. Although the specific drug tropatepine may not be widely available outside of France, we posit that it is reasonable to consider trials of other, more widely available, antimuscarinic agents commonly used in neurology, such as benztropine or trihexyphenidyl, in resistant cataplexy.

\section{Conclusion}

In this report of three narcoleptic patients, tropatepine was a potent treatment for severe, disabling and drug resistant cataplexy and may represent an efficacious and cost-effective treatment for drug resistant cataplexy, as well as other features of REM sleep dysfunction commonly observed in narcolepsy. The success of this treatment approach also reemphasizes the role of cholinergic mechanisms in the genesis of cataplexy, and may constitute a rationale for trials of other anticholinergic agents, such as benztropine or trihexyphenidyl, in countries where tropatepine is unavailable. 


\section{References}

1. Bassetti CLA, Adamantidis A, Burdakov D, et al. Narcolepsy - clinical spectrum, aetiopathophysiology, diagnosis and treatment. Nat Rev Neurol. 2019;15(9):519-539.

2. Morgenthaler TI, Kapur VK, Brown T, et al. Practice parameters for the treatment of narcolepsy and other hypersomnias of central origin. Sleep. 2007;30(12):1705-1711.

3. Lambert A, dachary JM, Marie C, et al. [A new synthetic antiparkinsonian drug, tropatepine hydrochloride in extrapyramidal syndromes induced by neuroleptics]. Encephale. 1976;2(2):115-121.

4. Lecendreux M, Maret S, Bassetti C, Mouren MC, Tafti M. Clinical efficacy of high-dose intravenous immunoglobulins near the onset of narcolepsy in a 10-year-old boy. J Sleep Res. 2003;12(4):347-348.

5. Vanni-Mercier G, Sakai K, Lin JS, Jouvet M. Carbachol microinjections in the mediodorsal pontine tegmentum are unable to induce paradoxical sleep after caudal pontine and prebulbar transections in the cat. Neuroscience Letters. 1991;130(1):41-45.

6. Grace KP, Horner RL. Evaluating the Evidence Surrounding Pontine Cholinergic Involvement in REM Sleep Generation. Frontiers in Neurology. 2015;6(190).

7. Niwa Y, Kanda GN, Yamada RG, et al. Muscarinic Acetylcholine Receptors Chrm1 and Chrm3 Are Essential for REM Sleep. Cell Reports. 2018;24(9):2231-2247.e2237.

8. Horner RL, Peever JH. Brain Circuitry Controlling Sleep and Wakefulness. Continuum (Minneap Minn). 2017;23(4, Sleep Neurology):955-972.

9. Nishino S, Mignot E. Pharmacological aspects of human and canine narcolepsy. Prog Neurobiol. 1997;52(1):27-78. 
10. Bun H, Monjanel- Mouterde S, Audebert C, Vincent Du Laurier M, Durand A, Cano JP. Pharmacokinetics of Tropatepine in Healthy Volunteers. Clinical Drug Investigation. 1997;14(1):53-60. 